\title{
Management of Hypertension in Patients with Cardiovascular Disease
}

\author{
${ }^{1} \mathrm{~K}$ Venugopal, ${ }^{2} \mathrm{Z}$ Sajan Ahmad
}

\begin{abstract}
Systemic hypertension (HTN) is the most common risk factor for cardiovascular disease (CVD) in the world. Management of HTN in established CVD requires an integrated approach that is built on the foundation of pathophysiology and tailored to the major patient subsets - stable ischemic heart disease (SIHD), acute coronary syndromes (ACS) and heart failure (HF). This review expands on these concepts by establishing the link between hypertension and the CVD subsets, discussing goals and targets in each situation, and finally looking at what the current guidelines recommend regarding the choice of pharmacotherapy.
\end{abstract}

Keywords: Acute coronary syndrome, Heart failure, Hypertension, Stable ischemic heart disease.

How to cite this article: Venugopal K, Ahmad ZS. Management of Hypertension in Patients with Cardiovascular Disease. Hypertens J 2016;2(1):10-15

\section{Source of support: Nil}

Conflict of interest: None

\section{INTRODUCTION}

Hypertension (HTN) is the most common risk factor for cardiovascular disease (CVD) in the world, particularly so in South Asia as reported by the Global Burden of Disease Study. ${ }^{1}$ The INTERHEART study across 52 countries demonstrated that nine risk factors were responsible for the development of acute myocardial infarction (MI) all over the world. ${ }^{2}$ The most important and frequent risk factor identified among these was systemic HTN. It has been shown that reduction in blood pressure $(\mathrm{BP})$ would result in significant reduction in the occurrence of coronary artery disease (CAD), stroke, and renal failure. Recent guidelines have modified the targets for BP lowering in elderly patients. Management of HTN in patients with cardiovascular problems, from primary and secondary prevention measures to control of BP in specific clinical situations such as stable ischemic heart disease, acute coronary syndromes (ACS), and heart failure (HF), needs to be critically assessed with respect to targets of $\mathrm{BP}$, specific pharmacotherapy in different

\footnotetext{
${ }^{1}$ Professor and Head, ${ }^{2}$ Assistant Professor

1,2 Department of Cardiology, Pushpagiri Medical College Thiruvalla, Kerala, India
}

Corresponding Author: K Venugopal, Professor and Head Department of Cardiology, Pushpagiri Medical College Thiruvalla, Kerala, India, Phone: +919847005444, e-mail: drkvenugopal@hotmail.com situations and maintaining optimal tissue perfusion especially in the coronary and cerebral circulation.

\section{Prevalence of Hypertension in India}

A meta-analysis of prevalence, awareness, and control of HTN in India published in 2014 showed an overall prevalence of $33 \%$ in urban and $25 \%$ in rural population. Only $25.3 \%$ of the rural population and $42 \%$ of urban population are aware of HTN and $25.1 \%$ of rural and $37.6 \%$ urban population are getting treatment. Control of BP was seen in $10.7 \%$ of rural Indians and $20.2 \%$ of urban Indians. ${ }^{3}$ As nearly $18 \%$ of the world population is from India, this would translate into vast numbers of potentially treatable patients who would benefit by prevention of premature CAD, stroke, and renal disease. It is estimated that HTN is directly responsible for $57 \%$ of all stroke deaths and $24 \%$ of coronary deaths in India. ${ }^{4}$

\section{Secondary Prevention in Patients with Hypertension and Coronary Artery Disease}

It is now clearly recognized that the lowering of $\mathrm{BP}$ is more important in long-term prognosis than the class of drugs used. The independent risk factors of CVD risk other than HTN are diabetes mellitus (DM), dyslipidemia, smoking, obesity, and chronic kidney disease (CKD). Presence of other concomitant risk factors greatly increases the risk of cardiovascular morbidity and mortality. Control of other risk factors is equally important when managing patients with HTN and established CVD. Recent lipid guidelines advocate the use of only statins in the four high-risk groups and the use of other lipid-lowering drugs has not been shown to reduce CVD risk. ${ }^{5}$ Control of diabetes in a patient with established CAD and HTN is important in secondary prevention strategies. Smoking in hypertensive patients carry higher mortality than nonsmokers and the HTN is severe in smokers compared with nonsmokers. Weight reduction, regular physical activity, and salt restriction are other important strategies in secondary prevention in hypertensive patients with CAD. Chronic kidney disease and peripheral arterial disease (PAD) are important considerations in the treatment of patients with HTN and CAD.

Drug therapy for secondary prevention in hypertensive patients with CAD has been mainly focused around 
angiotensin-converting enzyme inhibitors/angiotensin receptor blockers (ACEIs/ARBs) and beta-blockers (BBs). Beta-blockers are drugs of choice in patients with CAD and angina and with left ventricular (LV) dysfunction. Angiotensin-converting enzyme inhibitors are indicated in all patients after an MI. The HOPE trial ${ }^{6}$ and EUROPA $\operatorname{trial}^{7}$ demonstrated the cardioprotective effects of ACEIs. Angiotensin receptor blockers are currently indicated in a situation where ACEIs cannot be given. It is to be noted that the combination of ACEIs and ARBs did not give an additional benefit and are not currently recommended together. Calcium channel blockers (CCBs), though effective antihypertensive and antianginal agents, are not routinely recommended in secondary prophylaxis due to lack of data showing effectiveness. They can be considered in situations wherein BBs cannot be used as in severe bronchospastic disease.

\section{Hypertension in Established Cardiovascular Disease}

Management of HTN in established CVD is discussed under the following situations:

- Hypertension in a patient with stable ischemic heart disease (SIHD);

- Hypertension in a patient with ACS;

- Hypertension in a patient with HF.

\section{Hypertension Management in Patients with SIHD}

\section{The link}

The relationship between HTN and SIHD can be considered under the following aspects:

- Increase in myocardial oxygen demand: Episodes of surges in HTN could lead to an increase in myocardial oxygen demand and anginal episodes.

- Acceleration of atherosclerosis: Hypertension is well known to accelerate the atherosclerotic process and produce newer lesions and worsen angina.

- Left ventricular hypertrophy: Left ventricular hypertrophy, as a result of longstanding HTN, can worsen angina due to increase in the myocardial oxygen demand and can also lead to sudden cardiac death.

- Aortic dissection: Hypertension is the most important cause of aortic dissection in older patients and frequently coexists in the setting of atherosclerotic aortic disease and obstructive CAD.

\section{Aims of Treatment}

The aims of treatment in hypertensive patients with chronic stable angina are relief of anginal symptoms, reduction of ischemic episodes and prevention of death,
MI, and stroke. The current target for BP control is $<140 / 90 \mathrm{~mm} \mathrm{Hg}$, though lower targets of $<130 / 80 \mathrm{~mm}$ $\mathrm{Hg}$ have been considered in the past. The role of specific drug groups is discussed as follows:

- Beta-blockers: The pharmacological properties of negative inotropism and chronotropism of BBs make them the ideal drugs for patients with HTN and angina. Cardioselective BBs without intrinsic sympathomimetic action are the agents that are being used now for this purpose. Sinoatrial blocks, atrioventricular (AV) blocks, and severe bronchospastic disease are major contraindications for their use. Peripheral arterial disease was considered a relative contraindication for their use, but a meta-analysis showed that there was no worsening of claudication. The ACC/AHA practice guidelines on $\mathrm{PAD} 2005^{8}$ recommend the use of BBs in this situation. The newer generation drugs such as nebivolol and bisoprolol may be more appropriate in view of their selective actions and vasodilator properties.

- Calcium channel blockers: Currently, CCBs are prescribed in HTN with stable angina when BBs are contraindicated or not tolerated. They can be combined with BBs if the symptoms are not controlled with BBs or if the BP is remaining elevated. Dihydropyridine agents are preferably combined with BBs to avoid excess bradycardia. In patients with LV dysfunction, nondihydropyridine CCBs (verapamil and diltiazem) should not be used.

- ACEIs/ARBs: ACEIs are indicated in all CAD patients with stable angina and DM, LV dysfunction, or CKD if there are no contraindications. The current role for ARBs is in the same indications as ACEIs but when ACEIs are not tolerated. There is no clinical situation in $\mathrm{CAD}$ where ARBs have been found superior to ACEIs.

- Diuretics and nitrates: The ALLHAT trial demonstrated that diuretics can reduce cardiovascular events in hypertensive patients. ${ }^{9}$ Their role would mainly be supportive to BBs and ACEIs in hypertensive patients with CAD. Nitrates (long-acting) are useful in situations of angina when BBs or CCBs fail to control symptoms or are contraindicated.

\section{ACC/AHA/ASH Recommendations 2015}

Patients with HTN and chronic stable angina/SIHD can be treated with a regimen on the basis of the following recommendations ${ }^{10}$ :

- Beta-blockers (prior MI), ACEI/ARB (prior MI, LV dysfunction, DM, CKD) and a thiazide diuretic (control of HTN), class 1 A level of evidence (LOE) A. 
- The same combination of drugs in the absence of above indications becomes class 11a (if there is no LV dysfunction) in SIHD.

- Nondihydropyridine CCBs can be used if BBs are not tolerated or contraindicated, class 11a LOE B.

- For control of angina or HTN, a nonhydropyridine CCB can be added to BB, ACEIs/ARBs/thiazide such as diuretic class 11a LOE B.

- The target BP is $<140 / 90 \mathrm{~mm} \mathrm{Hg}$ (class 1 LOE A) but a lower target of $<130 / 80 \mathrm{~mm} \mathrm{Hg}$ may be considered in patients with stroke, carotid artery disease, peripheral artery disease, and abdominal aortic aneurysm.

\section{Hypertension and Acute Coronary Syndromes}

\section{The link}

The relationship between systemic HTN and ACS can be considered from the following perspectives:

- Hypertension as a risk factor for ACS: Apart from being an established factor for CAD, accelerated HTN can be a trigger for plaque rupture and hence precipitate an ACS.

- ACS leading to surge in BP: A high BP in a patient with ACS may be related to pre-existing HTN or due to sympathetic activation and anxiety.

- Hypertension and bleeding risk: Uncontrolled HTN increases the risk of bleeding in a patient with ACS who is being treated with antiplatelet agents, anticoagulants, and/or thrombolytic agents. Further, access sites for coronary interventions may be a source of bleeding too. Traditionally, a BP of $>180 / 110 \mathrm{~mm} \mathrm{Hg}$ has been considered to be a relative contraindication for thrombolytic therapy in ST-elevation myocardial infarction (STEMI) due to a higher risk for intracerebral hemorrhage. ${ }^{11}$ Such situations usually warrant a reduction in BP (commonly with nitroglycerine infusion) before administration of the thrombolytic agent. In addition, transient lowering of BP may often accompany thrombolytic therapy with streptokinase.

- Hypertension and mechanical complications of STEMI: Systemic HTN is a risk factor for free wall rupture. On the contrary, patients with pre-existing HTN are less likely to develop interventricular septal rupture.

- Drug treatment: The initiation and titration of BBs, ACEIs, and nitrates in ACS patients is closely linked to the patient's BP.

- Aortic dissection: A hypertensive patient with aortic dissection may present with an ACS, especially with an inferior wall MI, due to right coronary artery involvement by the proximal aortic dissection flap.

\section{Blood Pressure Goal in ACS}

The therapeutic BP goal has not been established in ACS. A high BP increases myocardial oxygen demand. On the contrary, an excessively low diastolic BP may impair coronary perfusion. Thus, the BP management in ACS must incorporate this "demand-supply" relationship. Further, there can be a marked fluctuation in BP in the initial phase of an acute MI. Blood pressure should therefore be lowered slowly and gradually, with care taken to avoid a diastolic BP of $<60 \mathrm{~mm} \mathrm{Hg}$. Generally, a BP of $<140 / 90 \mathrm{~mm} \mathrm{Hg}$ is acceptable in a post-ACS patient who is hemodynamically stable. A BP goal of $<130 / 80$ $\mathrm{mm} \mathrm{Hg}$ at predischarge is a reasonable goal.

\section{Drug Therapy of Hypertension in ACS}

A brief approach to the use of specific drugs in the setting of ACS is given below, based on the ACC/AHA/ASH recommendations 2015:

- Nitroglycerin: Nitroglycerin is an extremely useful and commonly prescribed drug for the management of high BP in ACS patients, especially in the setting of pulmonary edema or ongoing angina. However, it should be cautiously used in inferior wall $\mathrm{MI}$ and is contraindicated in right ventricular MI. Patients at an increased risk of hypotension include the elderly, individuals who are volume depleted, or those have used sildenafil within 24 hours or tadalafil within 48 hours. $^{12}$ Nitrate tolerance is a problem that can occur within 24 hours of infusion.

- Beta-blockers: They are particularly useful in the ACS because of reduction in $\mathrm{BP}$, heart rate, and, therefore, myocardial oxygen demand. Unless contraindicated, they should be started in all ACS patients within 24 hours of onset of the index event. Cardioselective (beta 1) BBs without intrinsic sympathomimetic activity such as metoprolol and bisoprolol are generally preferred. Carvedilol has an additional alpha 1 blocking effect that helps lower the BP; however, its beta 2 antagonism makes it unfavorable in patients with reactive airway disease. ${ }^{13}$ Intravenous agents such as esmolol may be considered in case of severe HTN with ongoing ischemia or tachyarrhythmia.

- Calcium channel blockers: The nondihydropyridine CCBs (verapamil and diltiazem) may be given if BBs are contraindicated due to reactive airway disease. However, they are contraindicated in ACS with left ventricular dysfunction. Dihydropyridine CCBs effectively lower the BP in the setting of ACS and are recommended if $\mathrm{BP}$ is not controlled with $\mathrm{BBs}$ and ACEIs. They have a specific role in the management of vasospastic angina too. 
- ACEIs and ARBs: These drugs have an established role in ACS, regardless of the presence of systemic HTN. ACEIs are preferred as first-line agents, with ARBs being recommended as effective replacements in case of intolerance to ACEIs. The combination of these two classes of drugs is not recommended.

- Aldosterone antagonists: Eplerenone and spironolactone are extremely useful drugs in ACS with HF and diabetes. However, they are contraindicated in renal dysfunction (creatinine $>2.5 \mathrm{mg} / \mathrm{dl}$ in males and $>2.0 \mathrm{mg} / \mathrm{dl}$ in females) and hyperkalemia (serum potassium $>5 \mathrm{meq} / \mathrm{l}$ ), due to risk of hyperkalemia, especially if creatinine clearance is $<50 \mathrm{ml} / \mathrm{min}$ and along with ACEI/ARB therapy. ${ }^{14}$

- Diuretics: They are not primarily chosen for BP control in ACS. Loop diuretics are often preferred in ACS patients with HF and pulmonary venous congestion, especially if there is renal dysfunction. However, particular attention must be given to the avoidance of hypokalemia, which can be particularly proarrhythmic in the setting of ACS. ${ }^{15}$

\section{Hypertension and Heart Failure}

\section{The link}

The interactions between these two major cardiovascular disorders can be viewed from the following angles:

- Hypertension as a risk factor for HF: Systemic HTN is one of the major risk factors for HF, both in systolic $\mathrm{HF}$ and in $\mathrm{HF}$ with preserved ejection fraction. Acute HF with pulmonary edema can occur in accelerated HTN.

- Shared pathophysiology: Both these disorders share common pathophysiological pathways, including activation of the renin-angiotensin system, heightened sympathetic tone, elevated endothelin levels, reduced nitric oxide (NO) levels, and endothelial dysfunction.

- Shared environmental influence: A high salt intake can increase the BP and also lead to worsening of preexisting HF. Therefore, dietary salt restriction is an integral component of treatment of these conditions.

- Relation to atrial fibrillation (AF): Both HTN and $\mathrm{HF}$ are well-established risk factors for AF, being components of the well-established CHA2DS2-VASc scoring system.

- Relation to renal disease: Uncontrolled or resistant systemic HTN may be a result of or by itself be a reason for renal dysfunction. Similarly, both acute and chronic HF can lead to cardiorenal syndromes.

- Common pharmacotherapeutic options: Angiotensinconverting enzyme inhibitors angiotensin receptor blockers, BBs, and diuretics are established drugs for the treatment of both these conditions.

\section{Goal Blood Pressure in Heart Failure}

In HF with reduced ejection fraction, the goal BP may be the "lowest reasonable" value that is not associated with symptoms of hypotension and/or evidence of hypoperfusion (e.g., prerenal azotemia). The achievement of the standard BP goal in the general population should not hinder the uptitration of HF medications to the guideline-directed doses, as these drugs have favorable survival benefits in HF that are independent of their BPlowering effects. Generally, the goal BP is $<140 / 90 \mathrm{~mm}$ $\mathrm{Hg}$. However, consideration may be given to lowering it to $<130 / 80 \mathrm{~mm} \mathrm{Hg}$. In the octogenarians, a BP $<130 / 65$ $\mathrm{mm} \mathrm{Hg}$ should be avoided and special care must be taken to avoid orthostatic hypotension.

\section{Drug Therapy in Hypertension and Heart Failure}

Specific issues pertaining to drug therapy of HTN in HF and vice versa are discussed as follows, based on the ACC/AHA/ASH recommendations 2015:

- Beta-blockers: The three major BBs that have proven benefit in HF are carvedilol, extended-release metoprolol, and bisoprolol. ${ }^{16,17}$ The additional alpha receptor blocking effect of carvedilol might theoretically provide an advantage in HTN. Nebivolol, with its NO donating property, might also be useful. ${ }^{18}$ Coadministration of BBs with digoxin and amiodarone is common in the setting of HF, especially in patients with atrial fibrillation. In such patients, in addition to heart rate, the electrocardiogram should be monitored for possible additive effects on the AV node.

- ACEIs and ARBs: In HF, the commonly used ACEIs are enalapril, ramipril, lisinopril, and trandolapril, and the major ARBs are losartan, valsartan, and candesartan. ${ }^{19,20}$ Combination of these two classes of drugs is discouraged due to higher incidence of adverse effects. Cardiorenal syndrome, with elevation of serum creatinine, may be an impediment to the use of these drugs in optimal doses. Among the ARBs used in the treatment of HTN, telmisartan has been noted to have a specific drug interaction with two drugs that are commonly prescribed in $\mathrm{HF}$ - digoxin and warfarin; the blood levels of these drugs may rise when coadministered with telmisartan. ${ }^{21}$

- Diuretics: Even though diuretics have not been found to improve survival in HF, their role is undeniable in controlling symptoms of HF and in reducing hospitalization rates. Chlorthalidone and hydrochlorothiazide, the major diuretics used in HTN, do not have an established role in HF. The coadministration of these drugs along with loop diuretics may be considered as part of "sequential nephron blockade" in patients with resistant HTN and/or HF with diuretic 
resistance. However, a higher incidence of electrolyte abnormalities should be anticipated.

- Calcium channel blockers: Verapamil and diltiazem are contraindicated in patients with systolic dysfunction. However, the dihydropyridine CCBs may be safely used in the treatment of HTN in HF patients. The renal safety and electrolyte neutrality are particularly helpful in patients with advanced HF. However, a small increase in heart rate may be anticipated.

- Aldosterone receptor antagonists: Spironolactone is a well-established component of the therapeutic armamentarium in systolic HF. In addition, it has been tested in diastolic HF and in resistant HTN.

- Drugs to avoid: Drugs to avoid in patients with HTN and HF with reduced ejection fraction are nondihydropyridine CCBs (such as verapamil and diltiazem), clonidine, moxonidine, and hydralazine without a nitrate. Nonsteroidal anti-inflammatory drugs should also be used with caution in this group, due to their effects on BP, volume status, and renal function.

\section{CONCLUSION}

The 2014 eighth Joint National Committee (JNC 8), while recommending a target $\mathrm{BP}$ for the general population of $<140 / 90 \mathrm{~mm} \mathrm{Hg}$ (for age $<60$ years) and $<150 / 90$ $\mathrm{mm} \mathrm{Hg}$ (for age $>60$ years), does not make any specific recommendation for patients with established CVD. The 2015 scientific statement from the AHA/ACC/ASH makes the following recommendations for target $\mathrm{BP}$ in patients with CAD: $<140 / 90 \mathrm{~mm} \mathrm{Hg}$ (weak recommendation), $<130 / 90 \mathrm{~mm} \mathrm{Hg}$ for some patients with CVD, such as those with $\mathrm{MI}$ or stroke (very weak recommendation), and $<150 / 90 \mathrm{~mm} \mathrm{Hg}$ for those over 80 years of age (weak recommendation). The SPRINT trial ${ }^{22}$ that has been published recently has shown that an intense BP control to targets $<120 / 80 \mathrm{~mm} \mathrm{Hg}$ compared with standard BP reduction $<140 / 90 \mathrm{~mm} \mathrm{Hg}$ reduced primary end points of MI, ACS, stroke, HF, hospitalization, or death from CVD but at a higher serious adverse events of hypotension, syncope, electrolyte imbalance, acute kidney injury, or renal failure. It appears as if the last word is yet to be written on target BP goals.

There remains no doubt regarding the fact that systemic HTN is a common and major risk factor for CVD and treating high BP is important in both primary and secondary prevention strategies. However, controversy still exists regarding the optimal target BP that balances the benefits and the risks, especially in specific patient subsets such as CAD, HF, stroke, and the elderly.

\section{REFERENCES}

1. Rodgers A, Lawes C, MacMahon S. Reducing the global burden of blood pressure-related cardiovascular disease. J Hypertens Suppl 2000 May;18(1):S3-S6.
2. Yusuf S, Hawken S, Ounpuu S, Dans T, Avezum A, Lanas F, McQueen M, Budaj A, Pais P, Varigos J, et al. Effect of potentially modifiable risk factors associated with myocardial infarction in 52 countries (the INTERHEART study): casecontrol study. Lancet 2004 Sep 11-17;364(9438):937-952.

3. Anchala R, Kannuri NK, Pant H, Khan H, Franco OH, Di Angelantonio E, Prabhakaran D. Hypertension in India: a systematic review and meta-analysis of prevalence, awareness, and control of hypertension. J Hypertens 2014 Jun;32(6):1170-1177.

4. Gupta R. Trends in hypertension epidemiology in India. J Hum Hypertens 2004 Feb;18(2):73-78.

5. Stone NJ, Robinson JG, Lichtenstein AH, Merz NB, Blum CB, Eckel RH, Goldberg AC, Gordon D, Levy D, Lloyd-Jones DM, et al. 2013 ACC/AHA guidelines on the treatment of blood cholesterol to reduce atherosclerotic cardiovascular risk in adults. Circulation 2014;129:S1-S45.

6. Yusuf S, Sleight P, Pogue J, Bosch J, Davies R, Dagenais G. The Heart Outcomes Prevention Evaluation Study Investigators. Effects of an angiotensin-converting-enzyme inhibitor, ramipril, on cardiovascular events in high-risk patients. $\mathrm{N}$ Engl J Med 2000;342:145-153.7.

7. Fox KM. European trial on reduction of cardiac events with perindopril in stable coronary artery disease investigators. Efficacy of perindopril in reduction of cardiovascular events among patients with stable coronary artery disease: randomised, double-blind, placebo-controlled, multicentre trial (the EUROPA study). Lancet 2003 Sep 6;362(9386):782-788.

8. Hirsch AT, Haskal ZJ, Hertzer NR, Bakal CW, Creager MA, Halperin JL, Hiratzka LF, Murphy WR, Olin JW, Puschett JB, et al. ACC/AHA 2005 Practice Guidelines for the management of patients with peripheral arterial disease (lower extremity, renal, mesenteric, and abdominal aortic): a collaborative report from the American Association for Vascular Surgery/Society for Vascular Surgery, Society for Cardiovascular Angiography and Interventions, Society for Vascular Medicine and Biology, Society of Interventional Radiology, and the ACC/AHA Task Force on Practice Guidelines (Writing Committee to Develop Guidelines for the Management of Patients With Peripheral Arterial Disease): endorsed by the American Association of Cardiovascular and Pulmonary Rehabilitation; National Heart, Lung, and Blood Institute; Society for Vascular Nursing; TransAtlantic Inter-Society Consensus; and Vascular Disease Foundation. Circulation 2006 Mar 21;113(11):e463-e654.

9. ALLHAT Officers and Coordinators for the ALLHAT Collaborative Research Group. The Antihypertensive and LipidLowering Treatment to Prevent Heart Attack Trial. Major outcomes in high-risk hypertensive patients randomized to angiotensin-converting enzyme inhibitor or calcium channel blocker vs diuretic: The Antihypertensive and LipidLowering Treatment to Prevent Heart Attack Trial (ALLHAT). JAMA 2002 Dec 18;288(23):2981-2997.

10. Rosendorff C, Lackland DT, Allison M, Aronow WS, Black HR, Blumenthal RS, Cannon CP, de Lemos JA, Elliott WJ, Findeiss L, et al. Treatment of hypertension in patients with coronary artery disease. A scientific statement from the American Heart Association, American College of Cardiology, and American Society of Hypertension. J Am Coll Cardiol 2015;65(18):1998-2038.

11. O'Gara PT, Kushner FG, Ascheim DD, Casey Jr DE, Chung MK, de Lemos JA, Ettinger SM, Fang JC, Fesmire FM, Franklin 
BA, et al; ACCF/AHA Task Force. 2013 ACCF/AHA guideline for the management of ST-elevation myocardial infarction: executive summary. A report of the American College of Cardiology Foundation/American Heart Association Task Force on Practice Guidelines. Circulation 2013;127:529-555.

12. Anderson JL, Adams CD, Antman EM, Bridges CR, Califf RM, Casey DE Jr, Chavey WE 2nd, Fesmire FM, Hochman JS, Levin TN, et al. ACC/AHA 2007 guidelines for the management of patients with unstable angina/non-ST-elevation myocardial infarction: executive summary. A report of the American College of Cardiology/American Heart Association Task Force on Practice Guidelines (Writing Committee to Revise the 2002 Guidelines for The Management of Patients with Unstable Angina/Non-ST-Elevation Myocardial Infarction). Circulation 2007;116:803-877.

13. Antman EM, Anbe DT, Armstrong PW, Bates ER, Green LA, Hand M, Hochman JS, Krumholz HM, Kushner FG, Lamas GA, et al. ACC/AHA guidelines for the management of patients with ST-elevation myocardial infarction: executive summary. A report of the ACC/AHA Task Force on Practice Guidelines (Writing Committee to Revise the 1999 Guidelines for the Management of Patients With Acute Myocardial Infarction). Circulation 2004;110:588-636.

14. Lewington S, Clarke R, Qizilbash N, Peto R, Collins R. Prospective Studies Collaboration. Age-specific relevance of usual blood pressure to vascular mortality: a meta-analysis of individual data for one million adults in 61 prospective studies. Lancet 2002 Dec 14;360(9349):1903-1913.

15. Katz JN, Gore JM, Amin A, Anderson FA, Dasta JF, Ferguson JJ, Kleinschmidt K, Mayer SA, Multz AS, Peacock WF, et al. Practice patterns, outcomes, and end-organ dysfunction for patients with acute severe hypertension: the studying the treatment of acute hypertension (STAT) registry. Am Heart J 2009 Oct;158(4):599-606.e1.
16. Effect of metoprolol $\mathrm{CR} / \mathrm{XL}$ in chronic heart failure: Metoprolol CR/XL Randomised Intervention Trial in Congestive Heart Failure (MERIT-HF). Lancet 1999 Jun 12;353(9169): 2001-2007.

17. Packer M, Fowler MB, Roecker EB, Coats AJ, Katus HA, Krum H, Mohacsi P, Rouleau JL, Tendera M, Staiger C, et al. Effect of carvedilol on the morbidity of patients with severe chronic heart failure: results of the carvedilol prospective randomized cumulative survival (COPERNICUS) Study. Circulation 2002 Oct 22;106(17):2194-2199.

18. Flather MD, Shibata MC, Coats AJ, Van Veldhuisen DJ, Parkhomenko A, Borbola J, Cohen-Solal A, Dumitrascu D, Ferrari R, Lechat $\mathrm{P}$, et al. Randomized trial to determine the effect of nebivolol on mortality and cardiovascular hospital admission in elderly patients with heart failure (SENIORS). Eur Heart J 2005 Feb;26(3):215-225.

19. Effect of ramipril on mortality and morbidity of survivors of acute myocardial infarction with clinical evidence of heart failure. The Acute Infarction Ramipril Efficacy (AIRE) Study Investigators. Lancet 1993 Oct;342(8875):821-828.

20. Black HR, Elliott WJ. Hypertension: a companion to Braunwald's heart disease. 2ed. Philadelphia: Saunders; 2007.

21. Køber L, Torp-Pedersen C, Carlsen JE, Bagger H, Eliasen P, Lyngborg K, Videbaek J, Cole DS, Auclert L, Pauly NC. A clinical trial of the angiotensin-converting-enzyme inhibitor trandolapril in patients with left ventricular dysfunction after myocardial infarction. Trandolapril Cardiac Evaluation (TRACE) Study Group. N Engl J Med 1995 Dec 21;333(25):1670-1676.

22. Wright JT Jr, Williamson JD, Whelton PK, Snyder JK, Sink KM, Rocco MV, Reboussin DM, Rahman M, Oparil S, et al. SPRINT Research Group: A randomized trial of intensive versus standard blood-pressure control. N Eng J Med 2015 Nov 26;373(22):2103-2116. 